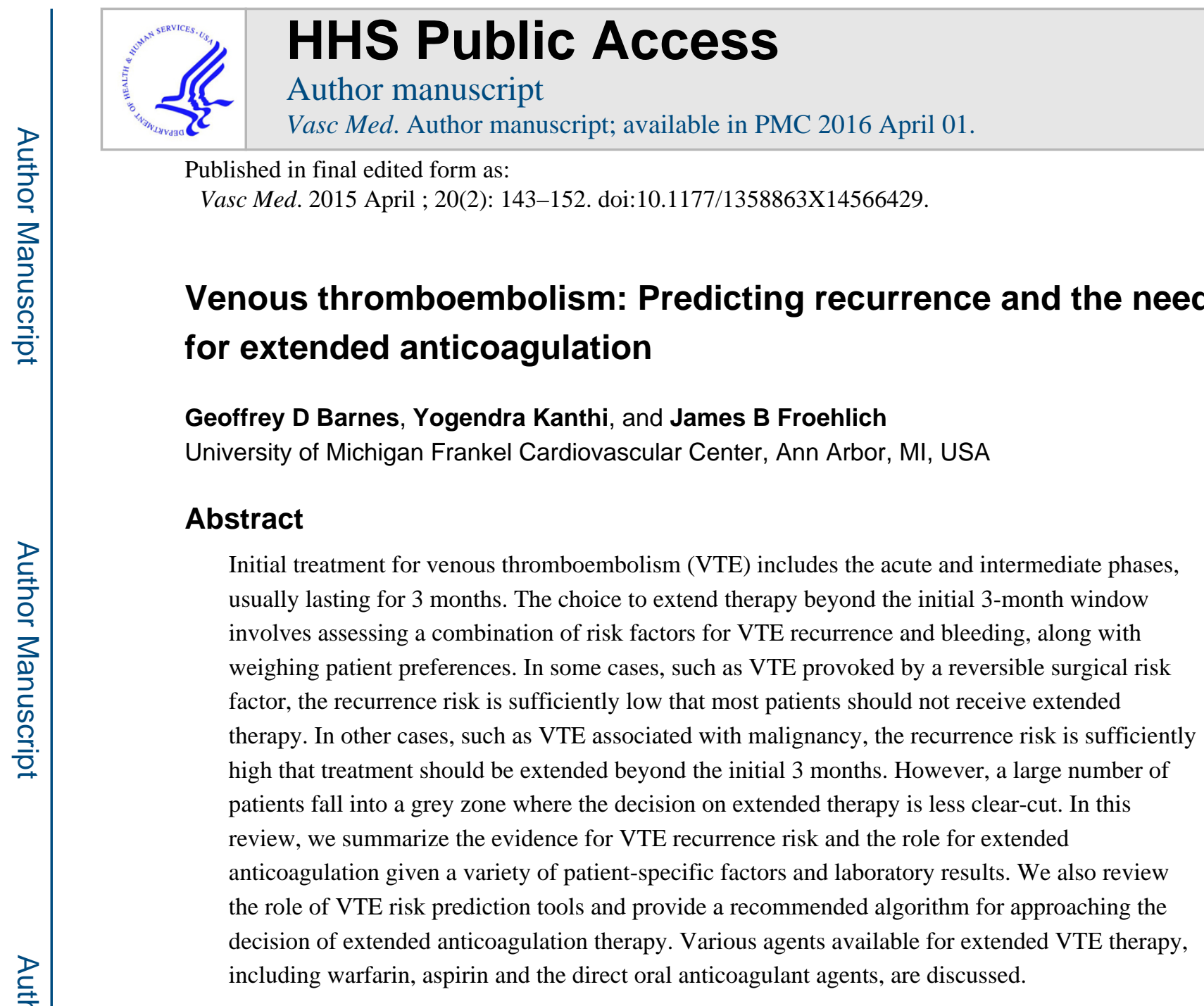

Keywords

Anticoagulation; venous thromboembolism

\title{
Background
}

Venous thromboembolism (VTE), which includes both deep venous thrombosis (DVT) and pulmonary embolism (PE), is a common medical disorder associated with significant morbidity and mortality. The annual incidence of VTE is estimated at 1-2 per 1000 population. ${ }^{1-3}$ VTE has a reported case fatality rate of $11.3 \%$ with a $30 \%$ risk of developing the post-thrombotic syndrome within 10-20 years following the incident VTE. ${ }^{1,4}$

While many practitioners consider VTE an acute illness, the effects of VTE are long-lasting and result in life-long vascular changes for many patients. After an initial event, recurrent

\footnotetext{
Reprints and permissions: sagepub.co.uk/journalsPermissions.nav

Corresponding author: Geoffrey Barnes, University of Michigan Frankel Cardiovascular Center, 2800 Plymouth Rd, Building 10, Room G016, Ann Arbor, MI 48109-2800, USA. gbarnes@umich.edu.

Declaration of conflicting interest

Dr Barnes has received research funding from Blue Cross Blue Shield of Michigan and BMS/Pfizer and consulting fees from Portola. Dr Froehlich has received research funding from Blue Cross Blue Shield of Michigan and BMS/Pfizer and consulting fees from Pfizer, Boehringer-Ingelheim, Merck, and Janssen pharmaceuticals. Dr Yogendra Kanthi has no conflict of interest to declare.
} 
VTE rates are reported to be as high as $17.5 \%$ at 2 years and $24.6 \%$ at 5 years. ${ }^{5}$ Interestingly, the risk of recurrence is highest in the initial year, declining thereafter but always with incremental risk. ${ }^{6}$ Additionally, the risk of recurrence once anticoagulation is discontinued does not appear to be significantly affected by the length of treatment. ${ }^{7}$

Treatment of VTE has been divided into three phases. The initial 'acute' phase of treatment is focused on halting the thrombotic process. ${ }^{8}$ Historically, this was achieved with heparinoid agents or fondaparinux, but recently a few of the newer direct oral anticoagulants (DOACs) have been shown to be equally effective in the acute phase (rivaroxaban and apixaban). The intermediate (long-term) phase usually lasts up to 3 months with a focus on reducing the risk of VTE recurrence. Previously, this was achieved with warfarin, but recently each of the available DOAC agents (dabigatran, rivaroxaban and apixaban) have been shown to be effective in this phase as well. The chronic (extended) phase focuses on secondary prevention of VTE recurrence ( $>3$ months after the initial event). There has been much debate about the need for extended treatment and the appropriate agent of choice in this phase, without general consensus.

In this review, we summarize the data for risk stratification of VTE recurrence, describe the published risk stratification tools and discuss risk factors for bleeding. We then summarize the data supporting the use of various antithrombotic agents for extended therapy. Finally, we provide a suggested approach to identifying appropriate patients for extended antithrombotic therapy following an initial VTE.

\section{Extended antithrombotic therapy}

Extended antithrombotic therapy refers to the secondary prevention of VTE with any anticoagulant or antiplatelet agent. This occurs after completion of the initial (acute) and intermediate (long-term) phases, usually 3 months following initiation of anticoagulation.

Current guidelines support the use of extended antithrombotic therapy in patients with high VTE recurrence risk or a known recurrent VTE as long as the bleeding risk is not prohibitively elevated. ${ }^{9}$ However, this is a Grade $2 \mathrm{~B}$ recommendation, defined as a weak recommendation with moderate quality evidence. ${ }^{10}$ The guidelines state that additional risk factors for VTE recurrence as well as patient preferences regarding the burden of anticoagulation may influence the decision to extend therapy in these situations. Many tools exist to predict the risk of VTE recurrence, which incorporate factors such as the situation in which a VTE developed, the location of the VTE, patient-specific aspects, as well as various laboratory and imaging tests. However, per the guidelines, a decision to extend therapy must incorporate a balance of the VTE recurrence risk with the risk for bleeding associated with anticoagulant therapy. ${ }^{9}$ Engaging patients in the decision-making process is a guidelineendorsed critical step. The development of shared decision-making tools is a much-needed area of research and development.

\section{Risk assessment for VTE recurrence}

The risk of VTE recurrence has been associated with a variety of factors. Many of these factors have been independently associated with VTE recurrence risk. However, not all 
factors have consistently been shown to predict recurrence or to benefit decision making in various populations.

\section{Type of initial VTE}

VTE episodes are categorized as provoked or unprovoked. Patients who develop VTE associated with a transient surgical procedure (surgically provoked VTE) have a very low rate of recurrence, estimated at $0.7 \%$ per year in the 2 years after stopping anticoagulation therapy. ${ }^{9,11}$ Patients who develop a non-surgical, provoked VTE (e.g. immobilization, pregnancy, use of estrogen-containing contraception) have a slightly higher recurrence risk, estimated at $4.2 \%$ per year in the same 2-year period after discontinuing anticoagulation therapy. ${ }^{11}$ Patients who develop VTE without a provoking factor have a higher rate of recurrence, estimated at $7.4 \%$ per year in the 2 years after discontinuing anticoagulation treatment. ${ }^{11}$ If VTE is associated with a known malignancy, recurrence rates are even higher. ${ }^{12}$ The role of other hypercoagulable states in VTE recurrence risk is discussed later.

\section{VTE location}

In an analysis of almost 3000 patients whose initial DVT was located in the distal lower extremity (calf DVT), recurrence rates were found to be lower than in patients with a more proximal location DVT (hazard ratio (HR) $0.49,95 \%$ confidence interval (CI) 0.34-0.71). ${ }^{13}$ However, in the same analysis, patients with a PE were found to have a similar recurrence risk as patients with proximal DVT (HR 1.19, 95\% CI 0.87-1.63). Nevertheless, multiple studies have shown that recurrent events in patients who have had a prior PE are more likely to be a second PE compared to patients with DVT. ${ }^{14,15}$ Additionally, patients with an initial $\mathrm{PE}$ have a risk of a recurrent, fatal PE that is two to three times higher than in patients with an initial DVT. ${ }^{4}$ In the three major trials of DOACs for extended therapy, there was no significant efficacy or recurrence difference between patients with an index PE versus an index DVT. ${ }^{16-18}$ Although many practitioners choose to treat patients with PE for longer durations than patients with a proximal DVT, the guidelines do not recommend different lengths of therapy.

\section{Patient factors}

A few patient-specific factors that are not modifiable play a role in the risk of VTE development and recurrence. A patient's age is a risk factor for both the development of VTE and the risk of recurrence. ${ }^{6,14,19}$ Male sex, for reasons that are not completely understood, is also a risk factor for VTE recurrence. ${ }^{20-23}$ However, the same increased risk for men is not consistently shown for an incident VTE. Multiple studies have demonstrated that men and women are at equal risk or women at slightly higher risk of developing an incident VTE. ${ }^{3}$

Cancer is a well-known risk factor for both the development of an initial and recurrent VTE. ${ }^{12}$ Recurrence rates without appropriate anticoagulation approach $21 \%$ in patients with an active malignancy over the first year. In patients with an active malignancy, use of lowmolecular-weight heparin (LMWH) is more effective than standard warfarin therapy in reducing VTE recurrence risk. ${ }^{24}$ 
Current investigation is ongoing into the role of systemic inflammatory conditions and the risk of VTE. In some studies, autoimmune disorders such as systemic lupus erythematosus and rheumatoid arthritis have been associated with a higher risk of VTE 25,26 and treatment with statins has been associated with a reduction in VTE occurrence, ${ }^{27,28}$ but further studies are necessary to identify a causative relationship and potential therapeutics.

\section{Laboratory studies}

Perhaps the most extensively studied laboratory measurement associated with VTE recurrence is the d-dimer. A number of studies have demonstrated that elevated levels of ddimer are associated with an increased risk of VTE recurrence. ${ }^{29-34}$ The majority of these studies have explored the role of d-dimer testing off anticoagulation, usually 1 month after completing warfarin therapy. A recent meta-analysis of seven studies exploring the predictive ability of a d-dimer checked 3 weeks to 2 months after stopping warfarin (minimum of 3 months of treatment) demonstrated that patients with a positive d-dimer had an annualized rate of VTE recurrence of $8.9 \%$ (95\% CI 5.8-11.9\%) compared to 3.5\% (95\% CI $2.7-4.3 \%$ ) in patients with a negative d-dimer. ${ }^{34}$ The utility of testing d-dimer while still on anticoagulation is less clear. In a recent study of 355 patients with idiopathic VTE, 28\% (95\% CI $21-31 \%$ ) of the patients with a normal d-dimer on anticoagulation developed an abnormal d-dimer at 1 month after cessation of anticoagulation therapy. ${ }^{35}$ Yet another study of 646 patients with a first, unprovoked VTE demonstrated that on-treatment d-dimer levels were highly predictive of VTE recurrence in women (relative risk (RR) 3.02, 95\% CI 1.416.51) but not for men (RR 1.14, 95\% CI 0.71-1.82). ${ }^{23}$ The combination of d-dimer testing with other clinical, laboratory and imaging tests as well as repeated d-dimer testing has also been explored. ${ }^{23,29-31,35-37}$

Hypercoagulable states are frequently tested for in patients with an incident VTE. However, their utility for predicting VTE recurrence or directing therapy is not endorsed routinely by guidelines, systematic reviews or expert opinion..$^{9,38-40}$ The one exception may be the antiphospholipid antibody syndrome, which is associated with significant VTE recurrence risk (RR 2) and has implications for treatment strategies. ${ }^{41,42}$ However, several parameters of antiphospholipid antibody testing may be altered by VTE and anticoagulation. ${ }^{43}$ The utility and yield of each test needs to be considered in each patient. Patients with factor V Leiden mutation are common (up to 5\% of all Caucasian patients). Many studies, but not all, have suggested a slightly elevated risk of VTE recurrence for patients with factor V Leiden mutations. ${ }^{4-46}$ However, the impact of this mutation and comparing recurrence risk with the risks of bleeding while on anticoagulation therapy make its clinical use less certain. Prothrombin mutations have generally not demonstrated elevated risk for VTE recurrence. ${ }^{42,44}$ Deficiencies of protein $\mathrm{C}$, protein $\mathrm{S}$ and antithrombin have been associated with VTE recurrence; however, the ability to test for these deficiencies while on warfarin therapy, as well as the rarity of these conditions, limits their clinical application. ${ }^{42,47}$ Elevated homocysteine levels have been associated with an increased risk of both arterial and venous thrombotic disorders, but the association with VTE recurrence is not well established and treatments targeted at reducing homocysteine levels have not been shown to reduce VTE risk. ${ }^{48-50}$ Patients with genetic dysfibrinogenemia are rare, but do appear to have an increased risk of VTE. ${ }^{51}$ While a combination of hypercoagulable states may 
increase VTE recurrence risk, this occurrence is rare. Expert opinion is generally not in favor of routine use of thrombophilia testing and many experts question the merit of testing for any VTE patients. ${ }^{46}$ For these reasons, we do not favor routinely ordering hypercoagulable testing in patients with a first VTE. Since patients with a recurrent VTE benefit from life-long therapy, there is usually no utility to performing hypercoagulable testing in this setting either. However, many practitioners find these tests useful in certain specific clinical situations, such as determining the need for VTE prophylaxis during pregnancy in women with a prior VTE.

\section{Mechanical features}

A number of studies have explored the role of persistent vascular occlusion and the associated risk of VTE recurrence. ${ }^{30,52} \mathrm{~A}$ recent meta-analysis demonstrated an elevated odds of VTE recurrence in DVT patients with residual vein thrombosis (odds ratio (OR) 2.5, 95\% CI 1.1-2.0) but not in the subset of patients with a first, unprovoked DVT (OR 1.25, 95\% CI 0.9-1.7). ${ }^{53}$ However, a subsequent patient-level meta-analysis found a weak association between residual vein thrombosis and VTE recurrence in patients with a first, unprovoked VTE (HR 1.32, 95\% CI 1.11-4.25). ${ }^{54}$

A recently published study examined the role of d-dimer testing in patients with residual vein thrombus after $3+$ months of anticoagulation. ${ }^{37}$ Patients with an elevated d-dimer while on anticoagulation therapy were maintained on treatment. Patients with a negative d-dimer had their anticoagulation stopped and a repeat d-dimer was performed sequentially at 15, 30, 60 and 90 days. Any elevated d-dimer level prompted re-initiation of anticoagulation therapy. During the 90 -day testing period, $52.3 \%$ of the patients enrolled maintained a negative d-dimer. VTE recurrence rates in patients treated with anticoagulation were significantly lower than in those who refused treatment despite an elevated d-dimer $(0.7 \%$ / 100 -patient-years vs $8.8 \% / 100$-patient-years, HR $2.92 ; p<0.001)$. VTE recurrence rates were $3 \% / 100$-patient-years in the group with persistently negative d-dimers and no anticoagulation treatment. We generally favor the use of d-dimer testing, but not duplex ultrasound testing for residual vein thrombus, in patients with an initial idiopathic VTE.

\section{VTE risk prediction tools}

Four risk prediction models have been developed to summarize key risk factors for VTE recurrence in a user-friendly tool (Table 1). The first tool published was the 'Men and HERDOO-2' score. ${ }^{23}$ In this study, 646 patients with a first, unprovoked VTE were followed over 4 years. Men were found to have an annualized recurrence risk of $13.7 \%$ (95\% CI 10.8-17.0\%). The risk of recurrence for women varied according to the presence of post-thrombotic syndrome, an elevated d-dimer (on anticoagulation) and obesity. For women with two or more of these findings, the annualized recurrence rate was $14.1 \%$ (95\% CI $10.9-17.3 \%$ compared to $1.6 \%$ (95\% CI $0.3-4.6 \%$ ) for women with one or fewer findings. Based on these results, the authors suggest that all men as well as women with two or more risk factors for VTE recurrence receive extended anticoagulation while women with zero or one risk factor are at sufficiently low risk of recurrence and do not require extended therapy. Limitations of this scoring system include the use of a d-dimer on anticoagulation and that approximately $75 \%$ of all patients are categorized as 'high risk' and therefore 
subject to extended anticoagulation. Still, this scoring system has clinical merit because it can be applied without having to stop anticoagulation before checking a d-dimer, a concern for many patients and practitioners. Validation of this scoring system and its utility in determining anticoagulation therapy is currently underway (NCT00967304).

The second tool developed was the Vienna risk model. ${ }^{20}$ In this study, 929 patients with a first, unprovoked VTE were followed for a median of 43.3 months after discontinuing anticoagulation. Recurrence risk was elevated in men (HR 1.9, 95\% CI 1.31-2.75), patients with proximal DVT (HR 2.09, 95\% CI 1.16-3.74) and PE (HR 2.60, 95\% CI 1.49-4.53) and elevated d-dimer (HR 1.27 per doubling, 95\% CI 1.08-1.51) when checked shortly after discontinuing warfarin. Unlike the 'Men and HERDOO-2' model, the Vienna risk model gives a range of VTE recurrence risks, not a binary high-versus low-risk state. While some practitioners may find this flexibility appealing, others might have difficulty determining what level of risk is sufficiently low to withhold extended anticoagulation. Additionally, the scoring system requires the use of a computer system or nomogram to appropriately calculate the risk based on d-dimer levels, limiting its ability to be applied from memory by practitioners in the clinic setting.

The third tool developed is the DASH model. ${ }^{22}$ In this study, 1818 patients with unprovoked VTE were examined after completion of anticoagulation therapy. An elevated d-dimer (checked 3-5 weeks after stopping anticoagulation), age <50 years, male sex and VTE not associated with hormone treatment (including estrogen-containing contraception and hormone replacement therapy) were found to be the main predictors of VTE recurrence. Two points are given for an elevated d-dimer: one point each for age $<50$ years and male sex, and two points subtracted if the VTE is associated with hormone therapy. The annualized rate of VTE recurrence was 3.1\% (95\% CI 2.3-3.9\%) for a score of one or less, $6.4 \%$ (95\% CI 4.8-7.9\%) for a score of two, and $12.3 \%$ (95\% CI 9.9-14.7\%) for a score of three or greater. The authors suggest that patients with a score of one or less are at sufficiently low risk of VTE recurrence that they should not be treated with extended therapy. This scoring system has the flexibility of estimating the recurrence rate for low, intermediate and high-risk patients as well as a suggested cut-off for extended therapy, but has not been validated in an external cohort or tested in a treatment-decision study. Also, some patients and practitioners may be uncomfortable using the d-dimer nearly 1 month after stopping anticoagulation to determine the need for extended therapy.

The final tool developed is the Louzada score. ${ }^{55}$ This score was developed in 543 patients with cancer-associated VTE and found that sex, primary tumor site, stage of the tumor and a history of prior VTE were independent predictors of VTE recurrence. One point is given to women, patients with lung cancer and patients with a prior VTE. One point is subtracted for breast cancer and two points are subtracted for a tumor TMN stage of I. In the derivation cohort, patients with a score of zero or less had a VTE recurrence frequency of $4.5 \%$, which the authors describe as low risk. In the validation cohort of 819 cancer-associated VTE patients, a score of -1 or less was associated with a VTE recurrence rate of $5.1 \%$ (low risk) and a score of zero was associated with a VTE recurrence rate of $9.9 \%$ (intermediate risk). While the score is designed for application in cancer-associated VTE patients and was externally validated, the variation in the scoring associated with a recurrence risk low 
enough for most practitioners to withhold extended anticoagulation limits its applicability at this time.

Each of the risk prediction tools has its merits, but uniform validation in external cohorts is still necessary. Additionally, while a risk prediction tool may be able to assess VTE recurrence risk, its ability to positively influence clinical decision making should not be assumed. For that reason, prospective testing of the clinical decision tool in a decisionmaking environment is advised to ensure that it is practical and patient-centered before widespread adoption of these tools.

\section{Bleeding risk assessment}

Similar to predicting VTE recurrence, predictors of bleeding while VTE patients are treated with anticoagulation exist. It is important to remember, however, that these predictors were identified in studies of patients receiving warfarin therapy, and may not carry the same association with bleeding for patients anticoagulated with DOACs. Significant predictors of bleeding include older age, prior bleeding, malignancy-associated VTE, renal or liver failure, thrombocytopenia and concurrent use of antiplatelet medications. Various attempts have been made to formalize a bleeding risk stratification tool for VTE patients. These include the OBRI, ${ }^{56}$ Kuijer, ${ }^{57}$ HEMORR $_{2}$ HAGES, 58 RIETE ${ }^{59}$, HAS-BLED,${ }^{60}$ ATRIA ${ }^{61}$ and $\mathrm{ACCP}^{9}$ scores. However, only the RIETE and Kuijer scores were developed specifically for acute VTE patients. The OBRI score was derived from an anticoagulated population of various indications for warfarin while the HEMORR 2 HAGES, HAS-BLED and ATRIA scores were initially tested in atrial fibrillation patients and only subsequently tested in VTE patients. The ACCP score includes elements common to multiple scoring systems and published literature, but was not formally tested when it was introduced. The risk elements summarized in Table 2 are found in many of these formal bleeding risk scores.

Use of bleeding risk assessments has not been widely adopted. This is likely due to each scoring system's limited predictive ability. While each score demonstrated modest predictive ability in its derivation and initial validation studies, similar success has not been seen in subsequent validation studies for VTE patients. In older adults, for whom bleeding risk is of significant concern, two validation studies found that no single scoring system had a c-statistic (area under the Receiver Operator Curve) greater than 0.61. ${ }^{62,63}$ Additionally, data have not been published detailing the bleeding risk factors for patients treated with the newer DOACs (e.g. dabigatran, rivaroxaban and apixaban).

The balance between VTE recurrence risk and bleeding risk is essential when patients and providers weigh the need for extended therapy, as is recommended by the guidelines. ${ }^{9}$ However, equating the occurrence of a single bleeding event to a recurrent VTE is not always appropriate. Therefore, clinicians must properly counsel patients to carefully consider the potential reversibility and downstream consequences of both a bleeding event and a recurrent VTE when making a decision to extend anticoagulation therapy. 


\section{Choice of antithrombotic agent}

Once a decision is made to extend treatment for secondary VTE prevention, a patient and provider must select the most appropriate antithrombotic agent. Historically, the only viable option was warfarin therapy. However, many newer options have been tested and approved by the FDA in recent years, specifically for secondary VTE prevention (Table 3). It is important to note that most of these studies compared a newer DOAC to a placebo control group.

\section{Warfarin}

Warfarin is typically given with a target INR range of 2.0-3.0. However, two pivotal studies examined the effectiveness of using lower target INR ranges for extended VTE treatment. In the first study, Ridker and colleagues compared low-intensity warfarin (INR target range 1.5-2.0) and placebo in 508 patients with idiopathic VTE who had received a median of 6.5 months of prior anticoagulation treatment. ${ }^{64}$ They showed that patients receiving lowintensity warfarin had a significantly reduced risk of VTE compared to patients treated with placebo (HR 0.36, 95\% CI 0.19-0.67) without a significant difference in major bleeding. This demonstrated that extended low-intensity therapy was superior to no therapy. However, in the second study, Kearon and colleagues tested the effectiveness of low-intensity warfarin (INR target range 1.5-1.9) versus standard intensity warfarin (INR target range 2.0-3.0) in 738 patients with unprovoked VTE who had completed $3+$ months of therapy. ${ }^{65}$ They demonstrated a higher risk of VTE recurrence (HR 2.8, 95\% CI 1.1-7.0) with no difference in major bleeding risk (HR 1.2, 95\% CI 0.4-3.0) in patients treated with low-intensity warfarin therapy. Based largely on these two trials, current guidelines recommend against the use of low-intensity warfarin therapy, preferring a standard INR target range of 2.0-3.0 for all patients being treated for VTE. ${ }^{9}$

\section{Low molecular weight heparin}

LMWHs are commonly used for the acute treatment of VTE, especially while warfarin therapy is being started. However, because of the need for injection, they are usually not the preferred agent for treatment after the initial hospitalization. They also have limitations in patients with severe renal impairment. One key exception is in patients with malignancyassociated VTE. In the CLOT trial, Lee and colleagues tested the effectiveness of LMWH against warfarin in 676 patients with an initial VTE and active cancer (Table 3). ${ }^{24}$ They found that treatment with LMWH was associated with a reduced risk of VTE recurrence compared to warfarin (HR 0.48, 95\% CI 0.30-0.77) with no added major bleeding risk. Patients in this trial most commonly had breast, colorectal, lung, genitourinary or gynecologic malignancies. Two-thirds of the patients had metastatic disease at enrolment. Based on this trial, the guidelines support the use of LMWH over warfarin in the setting of active cancer. ${ }^{9}$

\section{Dabigatran}

Dabigatran, an oral direct thrombin inhibitor, has been tested for both acute and extended therapy for VTE patients (Table 3). In the first of two trials for the extended therapy to prevent VTE recurrence, dabigatran $150 \mathrm{mg}$ twice a day was compared to placebo in 1343 
patients with a previously treated VTE after 6-18 months of therapy..$^{16}$ Patients treated with dabigatran had significantly reduced risk of VTE recurrence (HR $0.08,95 \%$ CI $0.02-0.25$ ) with an increased risk of major bleeding (HR 2.92, 95\% CI 1.52-5.60) compared to patients treated with placebo. In the second study, dabigatran $150 \mathrm{mg}$ twice a day was compared to warfarin in 2856 VTE patients previously treated for 3-12 months. ${ }^{16}$ There was no significant difference in VTE recurrence risk (HR 1.44, 95\% CI 0.78-2.64) or major bleeding (HR 0.52, 95\% CI 0.27-1.02) in the dabigatran versus warfarin treated groups. Dabigatran is not advised in VTE patients with an estimated creatinine clearance $₫ 3 \mathrm{ml} /$ min. ${ }^{16}$

\section{Rivaroxaban}

Rivaroxaban, an oral factor Xa inhibitor, has been tested for both acute and extended therapy for VTE patients (Table 3). In the EINSTEIN Extension trial, 1196 VTE patients who had completed 6-12 months of initial therapy were treated with either rivaroxaban 20 mg daily or placebo. ${ }^{18}$ Patients treated with rivaroxaban had a lower risk of VTE recurrence (HR 0.18, 95\% CI 0.09-0.39) with no significant difference in major bleeding compared to placebo. The EINSTEIN Choice trial is currently underway to compare rivaroxaban at 10 $\mathrm{mg}$ and $20 \mathrm{mg}$ to aspirin at $100 \mathrm{mg}$ for extended treatment of VTE and to assess the prevention of VTE with the risk of bleeding at standard and lower doses of rivaroxaban. Rivaroxaban is not advised in VTE patients with an estimated creatinine clearance $<30 \mathrm{ml} /$ $\min .^{18}$

\section{Apixaban}

Apixaban, another oral factor $\mathrm{Xa}$ inhibitor, has also undergone testing for both acute and extended VTE therapy (Table 3). In the AMPLIFY Extension trial, apixaban at two doses ( $2.5 \mathrm{mg}$ and $5 \mathrm{mg}$, both twice daily) were compared to placebo in 2486 VTE patients after an initial 6-12 months of anticoagulation therapy. ${ }^{66}$ Apixaban treated patients had a lower risk of VTE recurrence (RR $0.19,95 \%$ CI $0.11-0.33$ for $2.5 \mathrm{mg}$; RR $0.20,95 \%$ CI $0.11-0.34$ for $5 \mathrm{mg}$ ) without a significantly increased risk of bleeding compared to placebo. Apixaban is not advised in VTE patients with an estimated creatinine clearance $<25 \mathrm{ml} / \mathrm{min}^{66}$

\section{Aspirin}

Aspirin has historically been used to treat and prevent arterial thrombosis. However, recent investigation has explored its role in the prevention of venous thrombosis (Table 3). In the WARFASA trial, 402 patients with a first, unprovoked VTE who had completed 6-18 months of anticoagulant therapy were randomized to receive $100 \mathrm{mg}$ daily of aspirin versus placebo. ${ }^{67}$ Patients treated with aspirin had a reduced risk of VTE recurrence (HR 0.58, 95\% CI 0.36-0.93) without a difference in major bleeding event rates compared to patients receiving placebo. In the ASPIRE trial, 822 patients who had completed 6 weeks to 24 months of prior anticoagulation therapy for a first, unprovoked VTE were randomized to receive $100 \mathrm{mg}$ of aspirin or placebo. ${ }^{68}$ Aspirin therapy trended towards a reduced risk of VTE recurrence (HR 0.74, 95\% CI 0.52-1.05) without a difference in bleeding risk compared to patients who received placebo. 


\section{Choosing an appropriate agent}

Given the number of available treatment choices, it might be difficult for patients and practitioners to determine which form of extended therapy is most appropriate. Formal testing between the various agents would be of benefit to better inform patients of their treatment options. Current guidelines favor continuing to use the same agent in the extended treatment period as was used in the acute and intermediate phases. ${ }^{9}$ However, some patients may have a strong preference to change therapy. This is particularly true for patients who take warfarin for the acute and intermediate phase but wish to change to a non-monitored agent for extended therapy. For these patients, assessing their baseline renal function, ability to pay for brand-name agents and preferences for once daily vs twice daily administration can help to identify a preferred agent. For example, patients with poor renal function should not be prescribed dabigatran or rivaroxaban. Patients who have cost constraints may find that warfarin or aspirin are preferred agents. Patients who will find twice a day dosing difficult to comply with should avoid the use of dabigatran and apixaban. In patients for whom adherence is a major concern, the ability to monitor warfarin and its longer half-life may be beneficial. In patients with high bleeding risk, use of aspirin $81 \mathrm{mg}$, rivaroxaban or apixaban may offer the best bleeding-risk profile.

Engaging in a shared decision-making effort is critical to best identify any patient preferences, to review the risks and benefits, and to determine the optimal agent for extended therapy. However, it remains unclear what impact shared decision making would have on a patient's long-term adherence to anticoagulation therapy.

\section{Our approach}

Our approach to VTE treatment is summarized in Figure 1. In most patients with a first episode of VTE, we determine if the VTE was clearly provoked or associated with an active malignancy. Patients with a temporary provoking factor are treated for 3 months and then followed clinically without further testing. Patients with malignancy are treated with LMWH until the malignancy is felt to be in remission, and then followed clinically for signs of recurrence. We engage all other patients in a shared decision-making conversation to assess their preferences. Patients without a strong preference for limited or extendedduration therapy are assessed using a combination of clinical factors and a d-dimer. If the ddimer is elevated despite active anticoagulation (and no other reason for elevation), we continue therapy for an additional 3-6 months. Otherwise we will discontinue therapy and re-check the d-dimer in 1 month. If the d-dimer is elevated at that time, we would favor reinitiating extended secondary preventative treatment, either with aspirin or an anticoagulant drug. We encourage most of our male patients to strongly consider extended therapy given their increased risk of recurrence. Ultimately, however, the decision to extend therapy and the choice of agent is made by both the patient and provider, as a team.

\section{Summary}

In summary, many patients with VTE are at a substantial risk of VTE recurrence. However, it is important to balance the bleeding risks associated with extended anticoagulant therapy against the reduction in VTE recurrence risk when determining the appropriate length of 
therapy. Additional factors, such as costs, concurrent medication use and patient preference are important determinants of appropriate therapy. Use of various clinical, laboratory and imaging tests may be helpful in specific patients to better estimate the recurrence risk, but only if the results of those tests would influence a patient's treatment decision.

\title{
Acknowledgments
}

\author{
Funding \\ Dr Barnes is funded on the NIH grant 2-T32-HL007853-16.
}

\section{References}

1. Institute of Medicine (IOM). The Surgeon General's Call to Action to Prevent Deep Vein Thrombosis and Pulmonary Embolism. Rockville, MD: Office of the Surgeon General (US); 2008.

2. Naess IA, Christiansen SC, Romundstad P, et al. Incidence and mortality of venous thrombosis: a population-based study. J Thromb Haemost. 2007; 5:692-699. [PubMed: 17367492]

3. White RH. The epidemiology of venous thromboembolism. Circulation. 2003; 107:I4-8. [PubMed: 12814979]

4. Carrier M, Le Gal G, Wells PS, et al. Systematic review: case-fatality rates of recurrent venous thromboembolism and major bleeding events among patients treated for venous thromboembolism. Ann Intern Med. 2010; 152:578-589. [PubMed: 20439576]

5. Prandoni P, Lensing AW, Cogo A, et al. The long-term clinical course of acute deep venous thrombosis. Ann Intern Med. 1996; 125:1-7. [PubMed: 8644983]

6. Heit JA, Mohr DN, Silverstein MD, et al. Predictors of recurrence after deep vein thrombosis and pulmonary embolism: a population-based cohort study. Arch Intern Med. 2000; 160:761-768. [PubMed: 10737275]

7. Campbell IA, Bentley DP, Prescott RJ, et al. Anticoagulation for three versus six months in patients with deep vein thrombosis or pulmonary embolism, or both: randomised trial. BMJ. 2007; 334:674. [PubMed: 17289685]

8. Goldhaber SZ, Bounameaux H. Pulmonary embolism and deep vein thrombosis. Lancet. 2012; 379:1835-1846. [PubMed: 22494827]

9. Kearon C, Akl EA, Comerota AJ, et al. Antithrombotic therapy for VTE disease: Antithrombotic Therapy and Prevention of Thrombosis, 9th ed: American College of Chest Physicians EvidenceBased Clinical Practice Guidelines. Chest. 2012; 141:e419S-494S. [PubMed: 22315268]

10. Guyatt GH, Norris SL, Schulman S, et al. Methodology for the development of antithrombotic therapy and prevention of thrombosis guidelines: Antithrombotic Therapy and Prevention of Thrombosis, 9th ed: American College of Chest Physicians Evidence-Based Clinical Practice Guidelines. Chest. 2012; 141:53S-70S. [PubMed: 22315256]

11. Iorio A, Kearon C, Filippucci E, et al. Risk of recurrence after a first episode of symptomatic venous thromboembolism provoked by a transient risk factor: a systematic review. Arch Intern Med. 2010; 170:1710-1716. [PubMed: 20975016]

12. Prandoni P, Lensing AW, Piccioli A, et al. Recurrent venous thromboembolism and bleeding complications during anticoagulant treatment in patients with cancer and venous thrombosis. Blood. 2002; 100:3484-3488. [PubMed: 12393647]

13. Boutitie F, Pinede L, Schulman S, et al. Influence of preceding length of anticoagulant treatment and initial presentation of venous thromboembolism on risk of recurrence after stopping treatment: analysis of individual participants' data from seven trials. BMJ. 2011; 342:d3036. [PubMed: 21610040]

14. Agnelli G, Prandoni P, Becattini C, et al. Extended oral anticoagulant therapy after a first episode of pulmonary embolism. Ann Intern Med. 2003; 139:19-25. [PubMed: 12834314] 
15. Agnelli G, Prandoni P, Santamaria MG, et al. Three months versus one year of oral anticoagulant therapy for idiopathic deep venous thrombosis. Warfarin Optimal Duration Italian Trial Investigators. N Engl J Med. 2001; 345:165-169. [PubMed: 11463010]

16. Schulman S, Kearon C, Kakkar AK, et al. Extended use of dabigatran, warfarin, or placebo in venous thromboembolism. N Engl J Med. 2013; 368:709-718. [PubMed: 23425163]

17. Agnelli G, Buller HR, Cohen A, et al. Apixaban for extended treatment of venous thromboembolism. N Engl J Med. 2013; 368:699-708. [PubMed: 23216615]

18. Bauersachs R, Berkowitz SD, Brenner B, et al. Oral rivar-oxaban for symptomatic venous thromboembolism. N Engl J Med. 2010; 363:2499-2510. [PubMed: 21128814]

19. Anderson FA Jr, Wheeler HB, Goldberg RJ, et al. A population-based perspective of the hospital incidence and case-fatality rates of deep vein thrombosis and pulmonary embolism. The Worcester DVT Study. Arch Intern Med. 1991; 151:933-938. [PubMed: 2025141]

20. Eichinger S, Heinze G, Jandeck LM, et al. Risk assessment of recurrence in patients with unprovoked deep vein thrombosis or pulmonary embolism: the Vienna prediction model. Circulation. 2010; 121:1630-1636. [PubMed: 20351233]

21. Kyrle PA, Minar E, Bialonczyk C, et al. The risk of recurrent venous thromboembolism in men and women. N Engl J Med. 2004; 350:2558-2563. [PubMed: 15201412]

22. Tosetto A, Iorio A, Marcucci M, et al. Predicting disease recurrence in patients with previous unprovoked venous thromboembolism: a proposed prediction score (DASH). J Thromb Haemost. 2012; 10:1019-1025. [PubMed: 22489957]

23. Rodger MA, Kahn SR, Wells PS, et al. Identifying unprovoked thromboembolism patients at low risk for recurrence who can discontinue anticoagulant therapy. CMAJ. 2008; 179:417-426. [PubMed: 18725614]

24. Lee AY, Levine MN, Baker RI, et al. Low-molecular-weight heparin versus a coumarin for the prevention of recurrent venous thromboembolism in patients with cancer. N Engl J Med. 2003; 349:146-153. [PubMed: 12853587]

25. Choi HK, Rho YH, Zhu Y, et al. The risk of pulmonary embolism and deep vein thrombosis in rheumatoid arthritis: a UK population-based outpatient cohort study. Ann Rheum Dis. 2013; 72:1182-1187. [PubMed: 22930596]

26. Chung WS, Lin CL, Chang SN, et al. Systemic lupus erythematosus increases the risks of deep vein thrombosis and pulmonary embolism: a nationwide cohort study. J Thromb Haemost. 2014; 12:452-458. [PubMed: 24472157]

27. Glynn RJ, Danielson E, Fonseca FA, et al. A randomized trial of rosuvastatin in the prevention of venous thromboembolism. N Engl J Med. 2009; 360:1851-1861. [PubMed: 19329822]

28. Rodriguez AL, Wojcik BM, Wrobleski SK, et al. Statins, inflammation and deep vein thrombosis: a systematic review. J Thromb Thrombolysis. 2012; 33:371-382. [PubMed: 22278047]

29. Cosmi B, Legnani C, Cini M, et al. D-dimer and factor VIII are independent risk factors for recurrence after anticoagulation withdrawal for a first idiopathic deep vein thrombosis. Thromb Res. 2008; 122:610-617. [PubMed: 18304616]

30. Cosmi B, Legnani C, Cini M, et al. D-dimer and residual vein obstruction as risk factors for recurrence during and after anticoagulation withdrawal in patients with a first episode of provoked deep-vein thrombosis. Thromb Haemost. 2011; 105:837-845. [PubMed: 21359409]

31. Cosmi B, Palareti G. Update on the predictive value of D-dimer in patients with idiopathic venous thromboembolism. Thromb Res. 2010; 125(Suppl 2):S62-65. [PubMed: 20434008]

32. Maestre A, Trujillo-Santos J, Visona A, et al. D-dimer levels and 90-day outcome in patients with acute pulmonary embolism with or without cancer. Thromb Res. 2014; 133:384-389. [PubMed: 24438941]

33. Palareti G, Cosmi B, Legnani C, et al. D-dimer testing to determine the duration of anticoagulation therapy. N Engl J Med. 2006; 355:1780-1789. [PubMed: 17065639]

34. Verhovsek M, Douketis JD, Yi Q, et al. Systematic review: D-dimer to predict recurrent disease after stopping anticoagulant therapy for unprovoked venous thromboembolism. Ann Intern Med. 2008; 149:481-490. W494. [PubMed: 18838728]

Vasc Med. Author manuscript; available in PMC 2016 April 01. 
35. Cosmi B, Legnani C, Tosetto A, et al. Usefulness of repeated D-dimer testing after stopping anticoagulation for a first episode of unprovoked venous thromboembolism: the PROLONG II prospective study. Blood. 2010; 115:481-488. [PubMed: 19965693]

36. Eichinger S, Heinze G, Kyrle PA. D-dimer levels over time and the risk of recurrent venous thromboembolism: an update of the Vienna prediction model. J Am Heart Assoc. 2014; 3:e000467. [PubMed: 24385451]

37. Palareti G, Cosmi B, Legnani C, et al. D-dimer to guide the duration of anticoagulation in patients with venous thromboembolism: a management study. Blood. 2014; 124:196-203. [PubMed: 24879813]

38. Wells PS, Forgie MA, Rodger MA. Treatment of venous thromboembolism. JAMA. 2014; 311:717-728. [PubMed: 24549552]

39. Ho WK, Hankey GJ, Quinlan DJ, et al. Risk of recurrent venous thromboembolism in patients with common thrombophilia: a systematic review. Arch Intern Med. 2006; 166:729-736. [PubMed: 16606808]

40. Evaluation of Genomic Applications in Practice and Prevention (EGAPP) Working Group. Recommendations from the EGAPP Working Group: routine testing for Factor V Leiden (R506Q) and prothrombin $(20210 \mathrm{G}>\mathrm{A})$ mutations in adults with a history of idiopathic venous thromboembolism and their adult family members. Genet Med. 2011; 13:67-76. [PubMed: 21150787]

41. Garcia D, Akl EA, Carr R, et al. Antiphospholipid antibodies and the risk of recurrence after a first episode of venous thromboembolism: a systematic review. Blood. 2013; 122:817-824. [PubMed: 23760616]

42. Piazza G. Thrombophilia testing, recurrent thrombosis, and women's health. Circulation. 2014; 130:283-287. [PubMed: 25024124]

43. Pengo V, Tripodi A, Reber G, et al. Update of the guidelines for lupus anticoagulant detection. Subcommittee on Lupus Anticoagulant/Antiphospholipid Antibody of the Scientific and Standardisation Committee of the International Society on Thrombosis and Haemostasis. J Thromb Haemost. 2009; 7:1737-1740. [PubMed: 19624461]

44. Christiansen SC, Cannegieter SC, Koster T, et al. Thrombophilia, clinical factors, and recurrent venous thrombotic events. JAMA. 2005; 293:2352-2361. [PubMed: 15900005]

45. Sveinsdottir SV, Saemundsson Y, Isma N, et al. Evaluation of recurrent venous thromboembolism in patients with Factor V Leiden mutation in heterozygous form. Thromb Res. 2012; 130:467-471. [PubMed: 22512897]

46. Middeldorp S. Is thrombophilia testing useful? Hematology Am Soc Hematol Educ Program. 2011; 2011:150-155. [PubMed: 22160027]

47. Brouwer JL, Lijfering WM, Ten Kate MK, et al. High long-term absolute risk of recurrent venous thromboembolism in patients with hereditary deficiencies of protein S, protein $\mathrm{C}$ or antithrombin. Thromb Haemost. 2009; 101:93-99. [PubMed: 19132194]

48. Den Heijer M, Blom HJ, Gerrits WB, et al. Is hyperhomocysteinaemia a risk factor for recurrent venous thrombosis? Lancet. 1995; 345:882-885. [PubMed: 7707810]

49. DenHeijer M, Koster T, Blom HJ, et al. Hyperhomocysteinemia as a risk factor for deep-vein thrombosis. N Engl J Med. 1996; 334:759-762. [PubMed: 8592549]

50. Ray JG, Kearon C, Yi Q, et al. Homocysteine-lowering therapy and risk for venous thromboembolism: a randomized trial. Ann Intern Med. 2007; 146:761-767. [PubMed: 17470822]

51. Haverkate F, Samama M. Familial dysfibrinogenemia and thrombophilia. Report on a study of the SSC Subcommittee on Fibrinogen. Thromb Haemost. 1995; 73:151-161. [PubMed: 7740487]

52. Cosmi B, Legnani C, Iorio A, et al. Residual venous obstruction, alone and in combination with Ddimer, as a risk factor for recurrence after anticoagulation withdrawal following a first idiopathic deep vein thrombosis in the prolong study. Eur J Vasc Endovasc Surg. 2010; 39:356-365. [PubMed: 20034816]

53. Carrier M, Rodger MA, Wells PS, et al. Residual vein obstruction to predict the risk of recurrent venous thromboembolism in patients with deep vein thrombosis: a systematic review and metaanalysis. J Thromb Haemost. 2011; 9:1119-1125. [PubMed: 21382171] 
54. Donadini MP, Ageno W, Antonucci E, et al. Prognostic significance of residual venous obstruction in patients with treated unprovoked deep vein thrombosis: a patient-level meta-analysis. Thromb Haemost. 2014; 111:172-179. [PubMed: 24154729]

55. Louzada ML, Carrier M, Lazo-Langner A, et al. Development of a clinical prediction rule for risk stratification of recurrent venous thromboembolism in patients with cancer-associated venous thromboembolism. Circulation. 2012; 126:448-454. [PubMed: 22679142]

56. Beyth RJ, Quinn LM, Landefeld CS. Prospective evaluation of an index for predicting the risk of major bleeding in outpatients treated with warfarin. Am J Med. 1998; 105:91-99. [PubMed: 9727814]

57. Kuijer PM, Hutten BA, Prins MH, et al. Prediction of the risk of bleeding during anticoagulant treatment for venous thromboembolism. Arch Intern Med. 1999; 159:457-460. [PubMed: 10074953]

58. Gage BF, Yan Y, Milligan PE, et al. Clinical classification schemes for predicting hemorrhage: results from the National Registry of Atrial Fibrillation (NRAF). Am Heart J. 2006; 151:713-719. [PubMed: 16504638]

59. Ruiz-Gimenez N, Suarez C, Gonzalez R, et al. Predictive variables for major bleeding events in patients presenting with documented acute venous thromboembolism. Findings from the RIETE Registry. Thromb Haemost. 2008; 100:26-31. [PubMed: 18612534]

60. Pisters R, Lane DA, Nieuwlaat R, et al. A novel user-friendly score (HAS-BLED) to assess 1-year risk of major bleeding in patients with atrial fibrillation: the Euro Heart Survey. Chest. 2010; 138:1093-1100. [PubMed: 20299623]

61. Fang MC, Go AS, Chang Y, et al. A new risk scheme to predict warfarin-associated hemorrhage: The ATRIA (Anticoagulation and Risk Factors in Atrial Fibrillation) Study. J Am Coll Cardiol. 2011; 58:395-401. [PubMed: 21757117]

62. Poli D, Antonucci E, Testa S, et al. The predictive ability of bleeding risk stratification models in very old patients on vitamin $\mathrm{K}$ antagonist treatment for venous thromboembolism: results of the prospective collaborative EPICA study. J Thromb Haemost. 2013; 11:1053-1058. [PubMed: 23578305]

63. Scherz N, Mean M, Limacher A, et al. Prospective, multi-center validation of prediction scores for major bleeding in elderly patients with venous thromboembolism. J Thromb Haemost. 2013; 11:435-443. [PubMed: 23279158]

64. Ridker PM, Goldhaber SZ, Danielson E, et al. Long-term, low-intensity warfarin therapy for the prevention of recurrent venous thromboembolism. N Engl J Med. 2003; 348:1425-1434. [PubMed: 12601075]

65. Kearon C, Ginsberg JS, Kovacs MJ, et al. Comparison of low-intensity warfarin therapy with conventional-intensity warfarin therapy for long-term prevention of recurrent venous thromboembolism. N Engl J Med. 2003; 349:631-639. [PubMed: 12917299]

66. Agnelli G, Buller HR, Cohen A, et al. Apixaban for extended treatment of venous thromboembolism. N Engl J Med. 2012; 368:699-708. [PubMed: 23216615]

67. Becattini C, Agnelli G, Schenone A, et al. Aspirin for preventing the recurrence of venous thromboembolism. N Engl J Med. 2012; 366:1959-1967. [PubMed: 22621626]

68. Brighton TA, Eikelboom JW, Mann K, et al. Low-dose aspirin for preventing recurrent venous thromboembolism. N Engl J Med. 2012; 367:1979-1987. [PubMed: 23121403] 


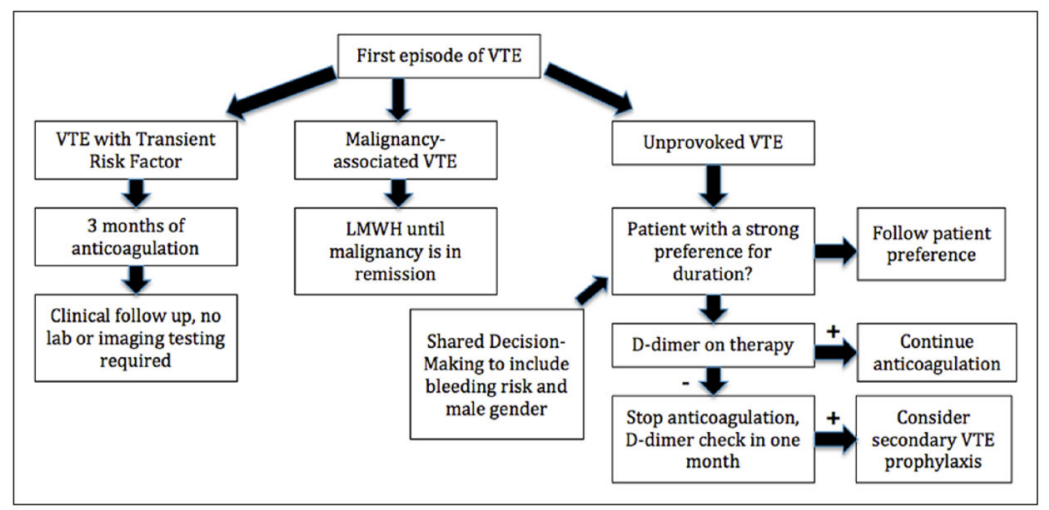

Figure 1.

Management of first venous thromboembolism. (VTE, venous thromboembolism; LMWH, low-molecular-weight heparin.) 
Table 1

Risk scoring algorithms for venous thromboembolism (VTE) recurrence.

\begin{tabular}{|c|c|c|c|c|}
\hline Prediction model & Elements & Points & Findings & \\
\hline \multirow[t]{8}{*}{ HERDOO- $2^{23}$} & Sex & NA & \multicolumn{2}{|l|}{ High risk: } \\
\hline & Signs of post-thrombotic syndrome & \multirow[t]{4}{*}{1} & \multirow{4}{*}{\multicolumn{2}{|c|}{$\begin{array}{ll}\text { - } & \text { Men }(13.7 \%, 95 \% \text { CI } 10.8-17 \%) \\
\text { - } & \text { Women with } \geq 2 \text { risk factors }(14.1 \%, 95 \% \text { CI } 10.9-17.3 \%) \\
& \text { Low risk: } \\
\text { - } & \text { Women with } 0-1 \text { risk factors }(1.6 \%, 95 \% \text { CI } 0.3-4.6 \%)\end{array}$}} \\
\hline & - Hyperpigmentation & & & \\
\hline & - $\quad$ Erythema & & & \\
\hline & - $\quad$ Redness & & & \\
\hline & Elevated d-dimer on anticoagulation & 1 & & \\
\hline & $\mathrm{BMI}>30 \mathrm{~kg} / \mathrm{m}^{2}$ & 1 & & \\
\hline & Age $\ 65$ & 1 & & \\
\hline \multirow[t]{4}{*}{ Vienna $^{20}$} & Male sex & NA & \multirow{4}{*}{\multicolumn{2}{|c|}{ Continuous HR $1.27-2.6$ based on nomogram }} \\
\hline & Proximal DVT & & & \\
\hline & $\mathrm{PE}$ & & & \\
\hline & $\begin{array}{l}\text { Elevated d-dimer ( } 3 \text { weeks post-VKA } \\
\text { discontinuation) }\end{array}$ & & & \\
\hline \multirow[t]{4}{*}{$\mathrm{DASH}^{22}$} & $\begin{array}{l}\text { Elevated d-dimer ( } 3-5 \text { weeks post- } \\
\text { anticoagulation discontinuation) }\end{array}$ & 2 & \multirow{4}{*}{\multicolumn{2}{|c|}{$\begin{array}{l}\text { Annualized recurrence risk: } \\
\begin{array}{cl}\text { - } & \leq \text { point }(3.1 \%, 95 \% \text { CI } 2.3-3.9 \%) \\
\text { - } & 2 \text { points }(6.4 \%, 95 \% \text { CI } 4.8-7.9 \%) \\
\text { - } & \geq 3 \text { points }(12.3 \%, 95 \% \text { CI } 9.9-14.7 \%)\end{array}\end{array}$}} \\
\hline & Age 50 & 1 & & \\
\hline & Male sex & 1 & & \\
\hline & Hormone replacement therapy & -2 & & \\
\hline \multirow[t]{5}{*}{ Louzada $^{55}$} & Female sex & 1 & \multicolumn{2}{|c|}{ Recurrence risk at 3-6 months: } \\
\hline & Prior VTE & 1 & • & Low risk: $\leq-1$ point $(5.1 \%)$ \\
\hline & Primary lung cancer & 1 & $\cdot$ & Intermediate: 0 points $(9.9 \%)$ \\
\hline & Primary breast cancer & -1 & $\cdot$ & High: $\geq 1$ point $(15.8 \%)$ \\
\hline & Tumor stage (TNM 1) & -2 & & \\
\hline
\end{tabular}

BMI, body mass index; HR, hazard ratio; DVT, deep venous thrombosis; PE, pulmonary embolism; VKA, vitamin-K antagonist; VTE, venous thromboembolism. 


\section{Table 2}

Predictors of bleeding risk in patients anticoagulated with warfarin for venous thromboembolism. ${ }^{9}$

- $\quad$ Older age $(>65-75)$

- $\quad$ Prior bleeding history

- Malignancy-associated venous thromboembolism (especially if metastatic)

- Renal insufficiency

- $\quad$ Liver failure/cirrhosis

- Diabetes

- Prior stroke

- Thrombocytopenia

- Anemia

- Concurrent use of antiplatelet agents

- $\quad$ Recent surgery

- Frequent falls

- Alcohol abuse

- Reduced functional capacity

- $\quad$ Poorly controlled warfarin therapy 


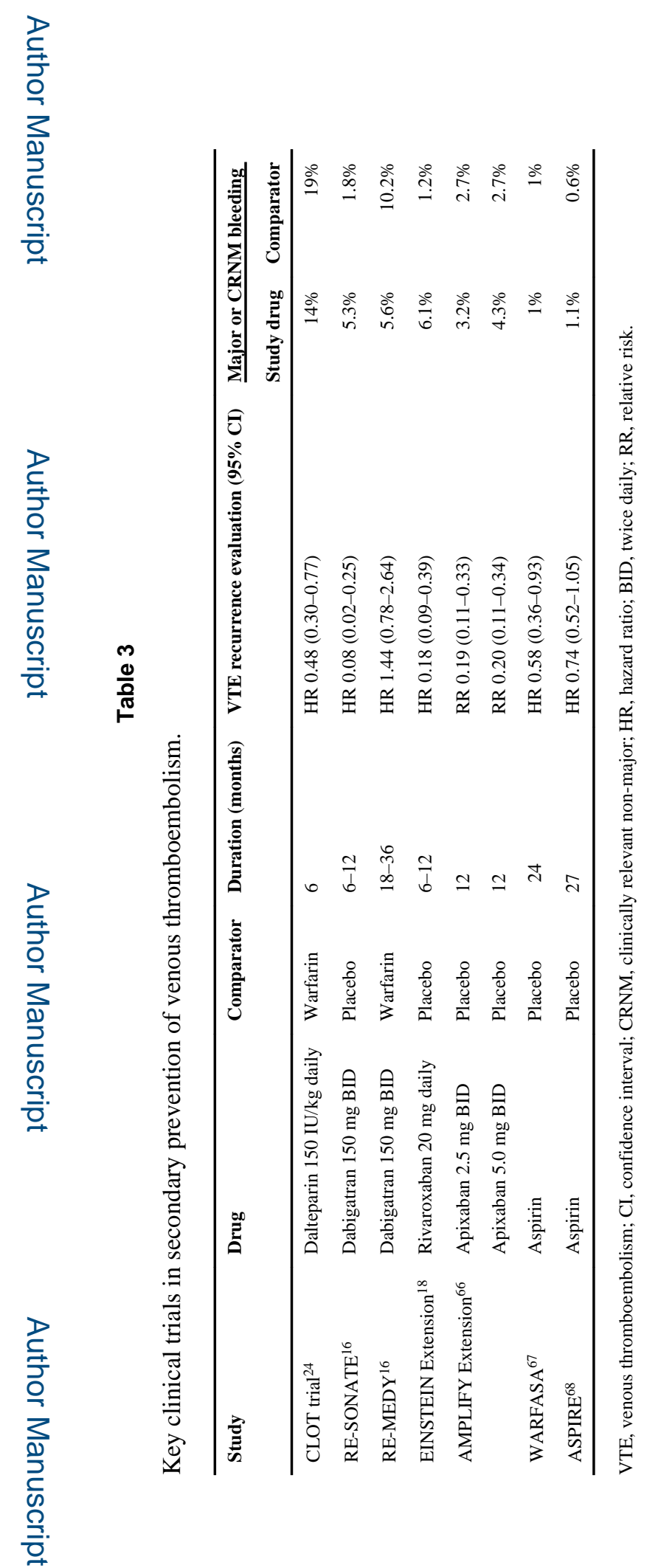

Vasc Med. Author manuscript; available in PMC 2016 April 01. 